\title{
Cognitive Radio: A New Standard in Wireless Communication Technology
}

\author{
R. Vadivelu and K. Sankaranarayanan
}

\begin{abstract}
The future of Wireless Communication Technology is going to be basically based on Cognitive Radio (CR) or Intelligent Radio (IR). At present the Spectrum allocation governed by the government agencies or the service providers who are in need of more spectrums, to manage the increasing frequency demands from the end users as the technological improvement increases exponentially. Efficient utilization of this scared Electromagnetic Radio Spectrum is the basis for the Cognitive Radio (CR). Spectrum Sensing being the fundamental issue of the Cognitive Radio i.e., detecting the underutilized spectrum of Primary User (PU) or Licensed User that can be efficiently utilized by the Secondary User (SU) or Unlicensed User. The spectrum is a scared natural resource, so it becomes essential to detect the unused spectrum of the PU to utilize it by other Users. The unused spectrums that are identified idle can be efficiently managed by spectrum management techniques. The managed spectrum should be mobilized for efficient utilization by the SUs. The spectrum mobilized can be shared properly by the SUs for proper usage which all governed by the physical layer operations of the Cognitive Radio. In this review paper, the fundamental issues of physical layer functions i.e., Spectrum Sensing, Spectrum Sharing, Spectrum Management and the NeXt generation Cognitive Radio functions have been analyzed and as a future work the higher layer issues of Cognitive Radio are considered to be the Link Layer, Network layer operations.
\end{abstract}

Keywords--- Cognitive Radio, Radio Resource, Spectrum Sensing, Spectrum Management, Spectrum Mobility, Spectrum Sharing

\section{INTRODUCTION}

$\mathrm{T}$ HE Electromagnetic Radio Spectrum resource requirement increased extremely as the Wireless Technology advancements increases exponentially. Basically, the Electromagnetic Radio Spectrum is allocated to the Licensed Users who are Primary Users. In a recent report [1] by Federal Communications Commission (FCC) of United States, states that the conventional fixed spectrum allocation rules formulated by the Governing Bodies have been resulted to have lesser efficiency, in using the spectrum bands as in Figure 1. Measurements in all over the world have been shows

R. Vadivelu, Assistant Professor, Department of Electronics and Communication Engineering, Sri Krishna College of Technology, Coimbatore, India, E-mail:vadiveluece@gmail.com

K. Sankaranarayanan, Dean, Easa College of Engineering and Technology, Coimbatore, India, E-mail:kkd_sankar@yahoo.com

DOI: 10.9756/BIJNTA.1501 the similar results. To overcome the spectrum scarcity, the new technology Cognitive Radio proposed first by Mitola in [2], is an emerging technique to fully exploit the underutilized radio spectrum. Simply, the introduction of this new Technology consists of basic challenges across the different layers of a Cognitive Radio system design like spectrum sensing, signal to noise interference management, resource allocation, RF design and Cognitive Radio implementation issues etc., which are physical layer parameters. There have been tremendous academic research towards Cognitive Radio $[3,4,5]$, defined as "an intelligent wireless communication system that is aware of its surrounding environment (i.e., outside world), and uses the methodology of understandingby-building to learn from the environment and adopt its internal states to statistical variations in the incoming $\mathrm{RF}$ stimuli by making corresponding changes in certain operating parameters (e.g., transmit-power, carrier-frequency, and modulation strategy etc.,) in real-time", with two fundamental objectives i.e.,

- Highly reliable communications whenever and wherever needed

- Efficient utilization of the radio spectrum.

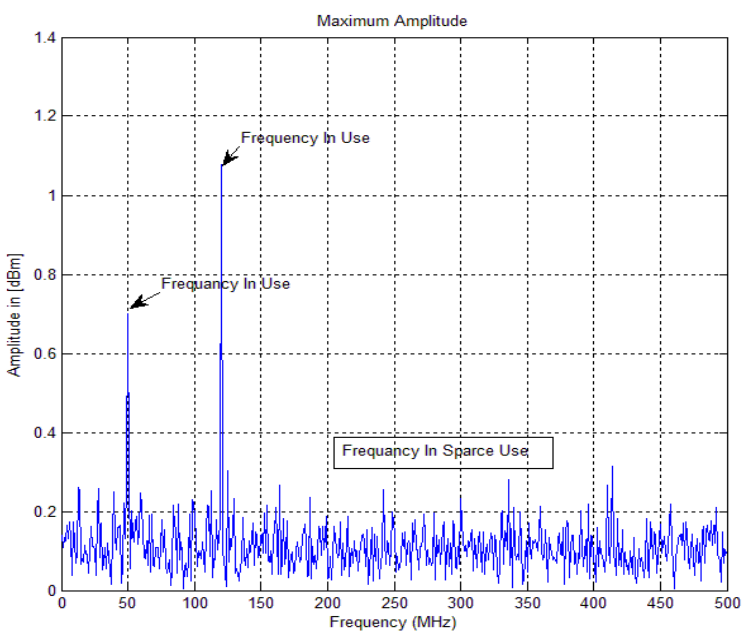

Figure 1: Frequency Usage

Wi-Max and the Wireless Innovation Alliance [6] including Google and Microsoft as members potentially estimated the so-called "White Spaces" Or "Spectrum Holes" in the Television (TV) spectrum as shown in Figure 2. The basic technology behind the Cognitive Radio is spectral estimation and efficient utilization of the spectrum which allows the secondary users to communicate over the spectrum allocated to the licensed users which are not utilizing it. The $\mathrm{SU}$ are required to frequently perform the operation of 
spectrum sensing that is detecting the presence or absence of the licensed users. If the PU utilizes the spectrum, it becomes active such that $\mathrm{SU}$ cannot utilize the spectrum. If $\mathrm{PU}$ is inactive then the SU can use the spectrum efficiently. The basic issues such as Orient, Plan, Learn, Decide, Act and Observe in Cognitive Radio Cycle are shown in Figure. 3 [7].

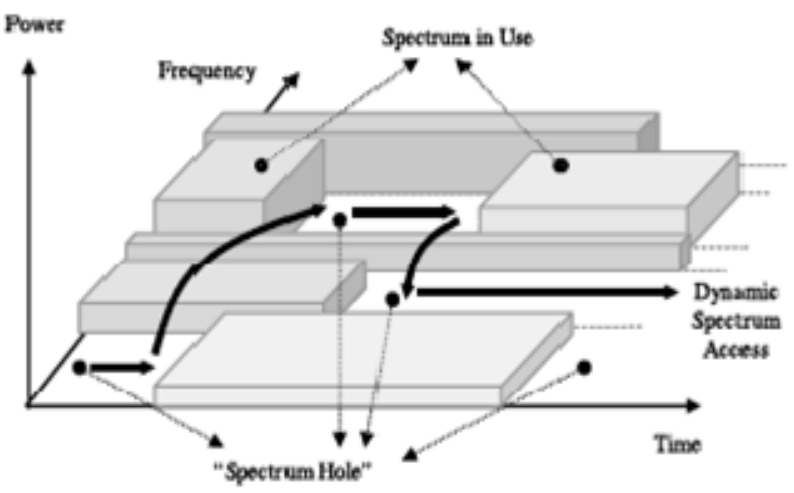

Figure 2: Spectrum Hole

The capabilities of CR technology can be classified into four main functionalities i.e., Spectrum Sensing, Spectrum Decision, Spectrum Sharing (SSH), and Spectrum Mobility (SM) or Spectrum Handoff (SH). Current research works concentrate on spectrum sensing, spectrum sharing and spectrum decision, but some few works on spectrum handoff have been done for Cognitive Radio Networks (CRN) especially in the case when transmissions are done over multiple channels in parallel.

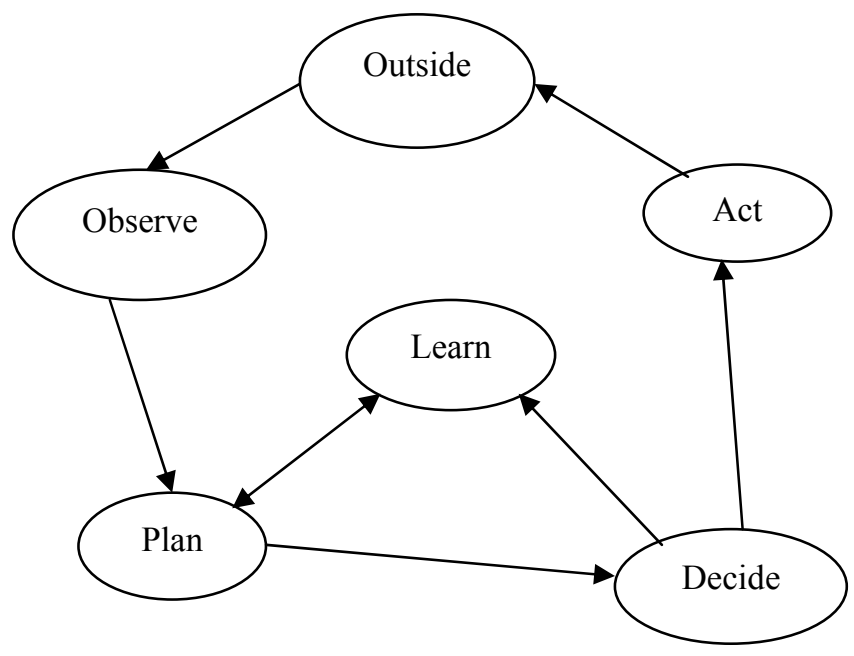

Figure 3: Cognitive Radio Cycle

This paper is organized as follows: Section II, deal with Spectrum Sensing Techniques in Section III Spectrum Management is dealt with. Spectrum Mobility is dealt in Section IV and in Section V Spectrum Sharing is discussed. NeXt generation Cognitive Radio is dealt in section VI and finally in section VII Concludes with future work.

\section{SPECTRUM SENSING}

Spectrum Sensing is the ability to sense the radio channel to find opportunities in spectrum and to adapt the radio parameters. Recent measurements show that the spectrum usage is mainly concentrated on certain portions of the spectrum, while majority of allocated spectrum varying from $15 \%$ to $85 \%$ was reported to be unutilized in the band below 3 $\mathrm{GHz}$ according to the Federal Communications Commission (FCC) Spectrum Policy Task Force [8] report. These spectrum holes or white spaces can be classified into (i) Black Spaces, which are occupied by high power interferes. (ii) Grey Spaces, which are low power interferes partially occupied. (iii) White Spaces, which are free. Spectrum Sensing is based on PU's Modulation type, Modulating Frequency, Modulating Power and other parameters. Spectrum Sensing is often considered as a spectrum detection technique. There are different Spectrum Sensing techniques were developed as shown in Figure 4 in order to detect the Spectrum Holes in the given Spectrum Band. Spectrum Detection characterized as Energy Detection is a parametric model [9] and feature detection [10] i.e., Cyclostationary feature detection is a Non Parametric Detection technique which are optimal way for high accuracy signal detection in wireless communication systems.

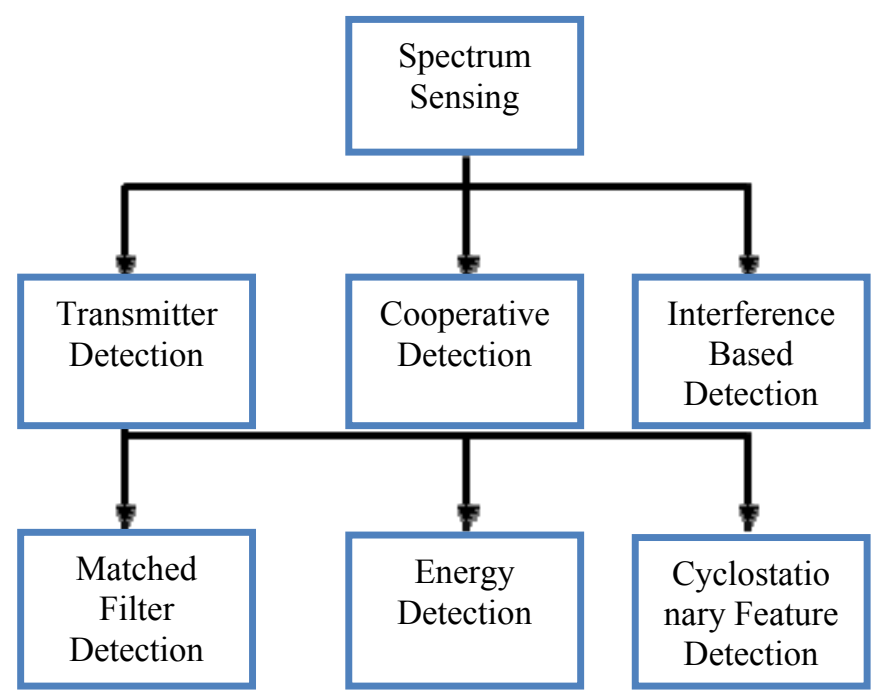

Figure 4: Spectrum Sensing Detection Techniques

Matched Filter (MF) technique has the advantage of requiring reduced time to achieve high processing gain due to coherency. Implementing this type of coherent detector is complex, since a SU would require extra hardware devoted to achieve carrier synchronization with each type of licensed PU. Moreover there may be cases in practice where MF is ruled out due to the lack of knowledge about PU in Real Time Spectrum Sensing. In [11] and [12] the compressive detectors using MF have been proposed. Energy Detection technique requires the knowledge of the PU signal which may not be available currently. In Energy Detection Technique, the presences of energy signals were determined without prior knowledge of PU. In Energy Detection (ED) technique the disadvantages are a) Signal to Noise Ratio (SNR) decision fixes the threshold value which changes with SNR. b) PUs signal and the interference signals both cannot be distinguished. c) Energy Detection technique fails if the signal power is spread over in large band. Advantage of wideband communication is that it can achieve high data rates by 
utilizing a large amount of spectrum but the disadvantage is the signals typically overlap each other [6]. Cyclostationary feature detection is used to extract signal features in the background of noise. Cyclostationary feature detection technique is a promising option for the Spectrum Sensing of Cognitive Radio, especially in the situation where Energy Detection is not so effective. Cyclostationary feature detection is a combination of Fast Fourier Transform (FFT) Accumulation and Spectral Correlation technique. It is suitable if ED technique fails. Disadvantage of Cyclostationary feature detection technique is that it requires complex computations and long observation time.

\section{SPECTRUM MANAGEMENT}

The best available spectrum captured from the channel used by the SU for communication without interfering the PU. Cognitive Radio should identify the best suitable spectrum band to meet the high Quality of Service (QoS) requirements over all the available spectrum bands, which results in efficient Spectrum Management. Spectrum Management can be classified based on the function as a) Spectrum Analysis and b) Spectrum Decision.

\section{A. Spectrum Analysis}

Spectrum Analysis is based on fixing appropriate sensing threshold value, to detect PU in the channel. To describe the dynamic nature of SUs, each spectrum hole should be characterized by considering not only the time-varying nature of SU, but also the nature of PU activity and the spectrum band parameters such as Channel Bandwidth (CB), Operating Frequency (OF) and Modulation Scheme (MS) etc., it is essential to define parameters such as Level of Interference (LoI), Path-Loss (PL), Channel Link Error (CLE) and Link Layer Delay (LLD) and Holding Time that can represent the quality of a particular Spectrum Band.

- $\quad$ Level of Interference (LoI): Some spectrum bands are more congested compared to others bands with respect to time. Hence, the spectrum band in use determines the Level of Interference characteristics of the channel. From the amount of the Level of Interference at the PU, the permissible power of a SU can be determined, which is the base for estimating the Channel Capacity.

- Path loss (PL): If the operating frequency increases, the PL increases. If the transmission power of the SU is same as that of PU and if the frequency is higher than its transmission power then the range decreases. If the transmission power is increased to compensate the path loss which results in higher interference for other users.

- Channel Link Errors (CLE): Channel Error rate changes depending on the scheme of modulation and the interference level of the spectrum band.

- Link Layer Delay (LLD): Link layer packet transmission delay increases due to different path loss, CLE and the level of interference since different types of link layer protocols are required at different spectrum bands.

- Holding Time (HT): The activities of PUs can affect the channel quality of the SU. Holding time is the time duration that the secondary users who can occupy PUs channel before getting interrupted. Quality would be increased if we increase the holding time. Holding time can decrease the spectrum handoff.

\section{B. Spectrum Decision}

Spectrum Decision is based on the need to meet the rules and regulations set out for radio spectrum access in International Telecommunication Union (ITU) Rules and Regulations and National Telecommunication Laws and Legislations (NTLL). Practical implementation issue of SM functions is more complex [8]. Once all available spectrum bands are characterized, SUs band has to be selected based on the Quality of Service (QoS) requirements such as Data Transfer Rate, Channel Error Rate, Packet Delay, Modulation Mode and the Bandwidth. Based on the decision rules framed to the spectrum band will be choosed [13]. In [14], few spectrum decision rules are proposed. An opportunistic frequency channel hopping protocol is proposed for the search of channel for better QoS, here the channel decision is based on SNR. In order to consider the PUs activity, the number of spectrum handoff either Hard Handoff $(\mathrm{HH})$ or Soft Handoff (SH), which happens in a certain spectrum bands, is used for spectrum decision [15].

\section{SPECTRUM MANAGEMENT}

The SUs are visitors in the Network Environment. The SUs can use the channel whenever the PUs not using the spectrum currently. If suppose the PUs enters the spectrum band suddenly, instantaneously the SUs have to vacate the spectrum band and can use any other vacant band in the spectrum, resulting in SM. That is, providing and maintaining a better spectrum opportunity SUs while it is switching from one band to other in the spectrum. SU uses the spectrum in a dynamic manner by allowing the radio terminals to operate in the best available frequency band, maintaining seamless communication requirements during the transition to better spectrum utilization. SM is the most important feature of a wireless communication system. Basically continuous service is required by supporting Handoff or handover from one cell to another by the Cognitive user. Handoff is the process of changing the channel functions such as Frequency, Time Slot and Code Spreading or combinations of these associated with the current Cognitive user while the user is in active condition. It is achieved either by crossing a cell boundary or by deterioration in quality of the signal in the current channel.

Handoff is divided into two broad categories Hard Handoff $(\mathrm{HH})$ and Soft Handoff $(\mathrm{SH})$ [16]. In $\mathrm{HH}$, the current resources are released before new resources are to be used. In $\mathrm{SH}$, both the current and new resources are used during the handoff course. However, poorly designed handoff schemes tend to generate very heavy signalling traffic and thereby an intense decrease in QoS. The reason why handoffs are precarious in wireless or mobile communication systems is that the neighbouring boundary cells are always using a disjoint subset of frequency bands. So negotiations must take place between the Mobile Station (MS) and the current serving 
Base Station (BS) and the next potential BS. Other related issues, such as Decision Making and Priority Strategies during overloading might contribute in the overall performance. In spectrum Handoff, a SU shift from occupied channel to free channel due to arrival of PU in the network.

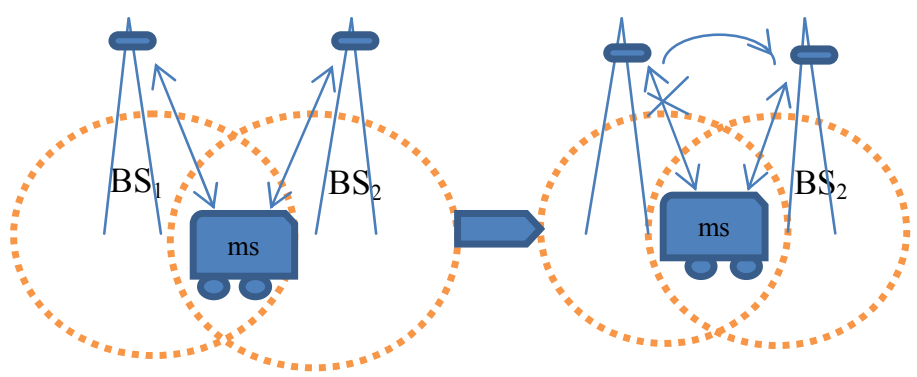

Figure 5: Before and After Handoff between BS and ms

Spectrum Handoff is required since the SU drops its connection due to mobility of SUs. The current spectrum band cannot provide good QoS requirement for the SU in the current band due to PU usage of the spectrum band. Re configurability property of the SU such as Operating Frequency, Modulation Scheme, Error Correction Code and Data rate etc., are adjusted. QoS is maintained for specific applications and are managed by the secondary user with reference with the PU. Figure 5 shows before and after Handoff between BS and MS.

\section{SPECTRUM SHARING}

The major challenge in the open spectrum practice is Spectrum Sharing, providing a fair spectrum to the SU using Spectrum Scheduling Algorithms (SSA) or methods. It can be regarded to be similar to generic Media Access Control (MAC) protocol problem, Carrier Sense Multiple Access (CSMA), Time Division Multiple Access (TDMA) or Frequency Division Multiple Access (FDMA) problem existing in communication systems as shown in Figure 6.

A CR is also characterized by its re-configurability; the ability of reconfiguring or changing its operating parameters for transmission without changing any hardware components. The re-configurable communication parameters of $\mathrm{CR}$ are such as carrier/operating frequency, modulation scheme, and transmission power and communication technology. Spectrum utilization efficiency further improved in a CR network by spectrum sharing. The PU always have a higher priority is needed over the SUs in utilizing the spectrum resources. So the fundamental challenge of spectrum sharing is to provide the best QoS to the PU, by keeping minimum interference by the SU. Therefore, it is crucial in the design of CR systems to take into consideration two main significant and conflicting objectives, i) maximizing the throughput of the cognitive system and ii) minimizing the interference to the primary user. In paper [17], [18] a capacity achieving transmit spatial spectrum for a single secondary link in a CR network under its own transmitter power constraint and interference power constraint at the primary receiver has been explained. The proposed problem was formulated as an optimization problem.

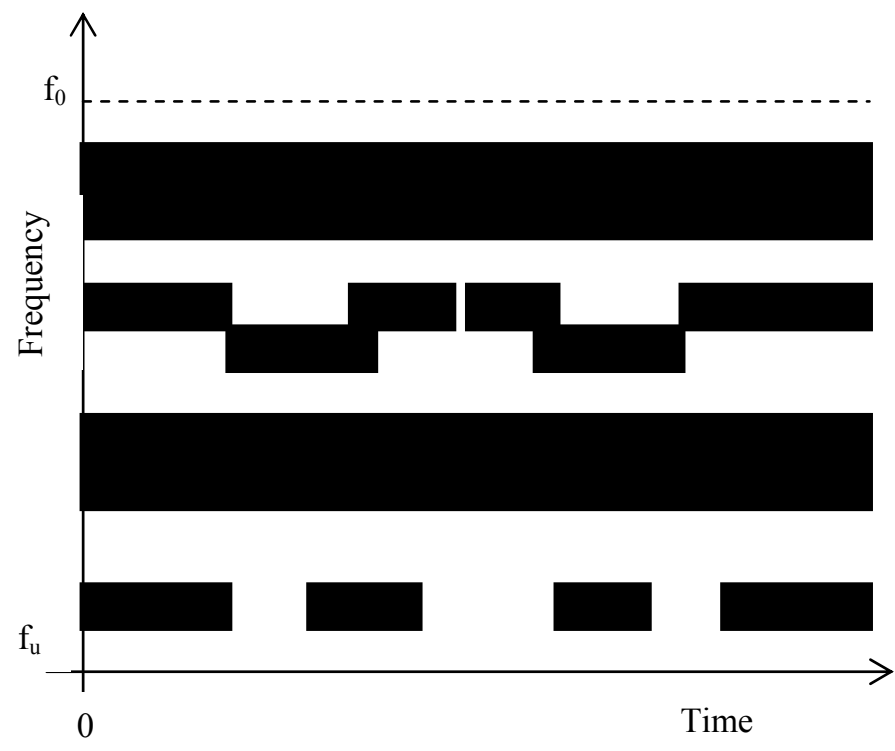

Figure 6: FDMA / TDMA Signals over the Time / Frequency Plane, Spectrum Pool

In [19] the problem of joint power control and beam forming in the downlink of the CR network was studied for users of limited numbers. Large number of cognitive users who are operating in the same frequency of PU uses a scheduling mechanism that uses the spectrum in efficient manor results in Opportunistic Spectrum Sharing.

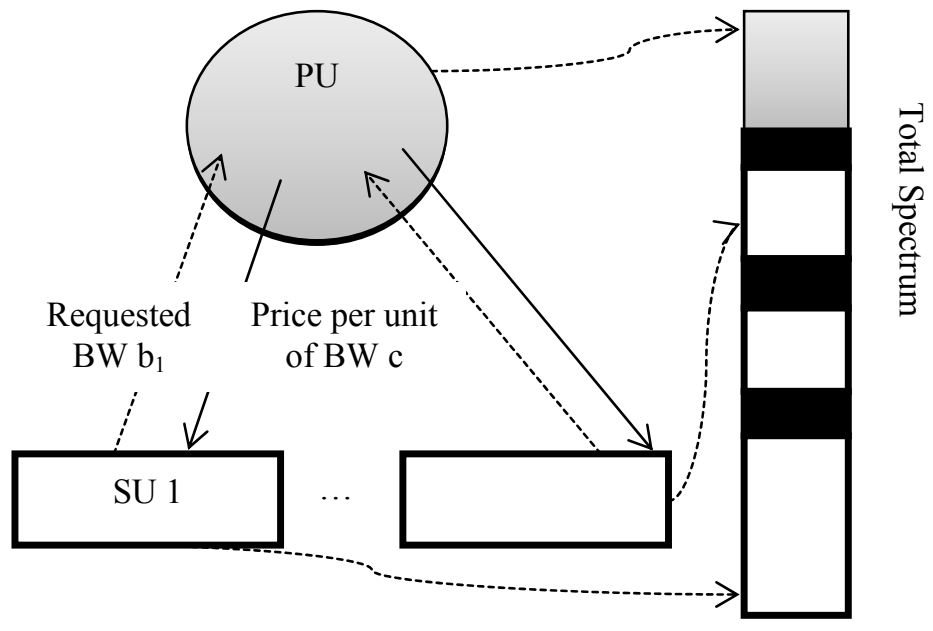

Figure 7: System Model for Spectrum Sharing

Advantage of opportunistic Spectrum Sharing is to maximize the sum rate throughput of the SU system and its inference avoidance with PU [20]. Optimal sum of spectrum sharing that is the number of SUs in the network with reference to PUs that maximizes the throughput in the network explained in [21]. Model for distributed dynamic strategy adaptation is proposed and the stability characteristics such as convergence, stability region, chaotic behaviour and sensitivity to initial strategies of the scheme are analysed and the system performance of the spectrum sharing model is evaluated under the system parameters such as radio channel 
quality, revenue function and learning rate for dynamic strategy adaptation [22]. Figure 7 shows the system model for spectrum sharing.

\section{NeXt GENERATION COGNITIVE RAdio}

Dynamic and Static spectrum sharing techniques are useful for designing NeXt Generation (xG) Cognitive Radio Systems. Functions of $x G$ network include such as Spectrum Management, Spectrum Mobility and Spectrum Sharing [23]. The Spectrum Sharing process is based on five major issues.

1. Spectrum Sensing (SS): An xG user can only allocate a portion of the spectrum if that portion is not used by an unlicensed user. Then the next node of the $\mathrm{xG}$ node aims to Transmit Packets, before transmit packets it needs to aware the spectrum usage around its vicinity.

2. Spectrum Allocation (SA): Based on the availability of spectrum, the node can allocate the channel. The spectrum allocation is not only based on spectrum availability, it is also determined based on internal policies. Hence, the design of a spectrum allocation policy is to improve the spectrum utilization performance.

3. Spectrum Access (SAc): Since there are more $x G$ nodes trying to access the spectrum, the access should be coordinated in order to prevent multiple users colliding in overlapping portions of the spectrum.

4. Transmitter-Receiver Handshake (TRH): After determining the spectrum for communication, the receiver of this communication should also be indicated about the selected spectrum. A transceiver handshake protocol is essential for efficient communication in $\mathrm{xG}$ networks.

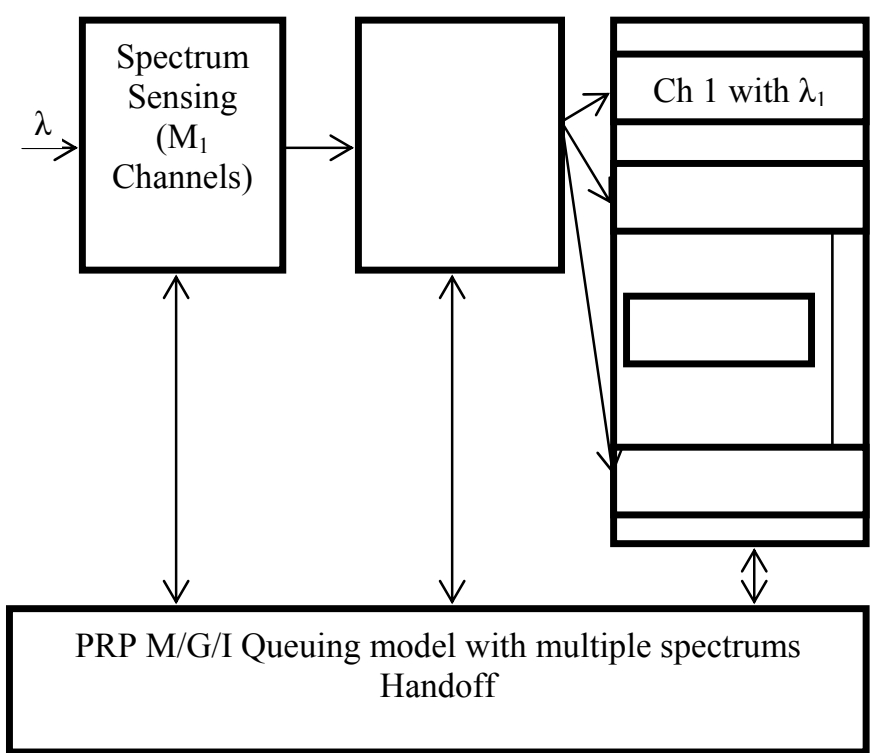

Figure 8: Relationship between Spectrum Sensing, Spectrum

Decision, Spectrum Sharing and Spectrum Mobility

5. Spectrum Mobility (SM): The specific portion of the spectrum allocated to a licensed user, the unlicensed user's communication needs to be continued in another vacant portion in the band. Hence, Spectrum Mobility is also important for successful communication between $\mathrm{xG}$ nodes.
Figure 8 shows the relationship between Spectrum Sensing, Spectrum Decision, Spectrum Mobility and Spectrum Sharing.

\section{CONCLUSION AND FUTURE WORKS}

Cognitive Radio Technology is the favourable future technique in wireless communication technology particularly, with dynamic cooperation of the spectrum-sharing process, significant "white space" can be created, which in turn, makes it possible to improve spectrum utilization under continually changing user environment. The dynamic spectrum-sharing capability is based on (a) Shift in wireless communications from transmitter to receiver, whereby interference power rather than transmitter emission is regulated. (b) Awareness of and adaptation to the environment by the radio. In this paper the fundamental issues related to physical layer operations of Cognitive Radio such as Spectrum Sensing, Spectrum Sharing and Spectrum Management were discussed. As a future work, the higher Cognitive layer operations such as Link Layer Operations responsible for Group Management, Link Management and Medium Access Control (MAC) can be analysed and implemented. Next Considering the Network Layer to increase the prospects of channel capacity, coexistence, compatibility, and interoperability.

\section{REFERENCES}

[1] Federal Communications Commission (FCC), "Spectrum Policy Task Force", Rep. ET Docket no. 02-135, Nov. 2002.

[2] J. Mitola, "Cognitive radio: An integrated agent architecture for software defined radio," Doctor of Technology, Royal Inst. Technol. (KTH), Stockholm, Sweden, 2000.

[3] M. A. Fischler and O. Firschein, Intelligence: "The Brain, and the Computer", Reading: Addison-Wesley, Pp. 81-83, 1987.

[4] B. Fette, "Technical challenges and opportunities," presented at the Conf. Cognitive Radio, Las Vegas, NV, March 15-16, 2004.

[5] J. Mitola, Ed., "Special issue on Software Radio," in IEEE Communication Mag., May 1995.

[6] Anuj Batra, Srinivas Lingam, Jaiganesh Balakrishnan, "Multi-band OFDM: A Cognitive Radio for UWB”, ISCAS 2006.

[7] I. F. Akyildiz, W. Lee, M. C. Vuran, and S. Mohanty, "NeXt generation dynamic spectrum access cognitive radio wireless networks: a survey," Computer Networks: The International Journal of Computer and Telecommunications Networking, Vol. 50, no. 13, Pp. 2127 - 2159, Sep. 2006.

[8] S. Haykin, "Cognitive Radio: Brain-Empowered Wireless Communications", IEEE Journal on Selected Area in Communications, Vol.23, no 2, Pp. 201-220, Feb. 2005.

[9] H. Urkowitz, "Energy detection of unknown deterministic signals", Proceeding of the IEEE, Vol. 55, No. 4, Pp. 523-531, Apr. 1967.

[10] M. Davenport, M. Wakin, and R. Baraniuk, "Detection and estimation with Compressive measurements", Rice ECE Department Technical Report TREE 0610, November 2006.

[11] J. Haupt and R. Nowak, "Compressive sampling for signal detection", IEEE Int. Conf. on Acoustics, Speech, and Signal Processing (ICASSP), Honolulu, Hawaii, April 2007.

[12] J. Zander and S. L. Kim, "Radio Resource Management for Wireless Networks", Norwood, MA: Artech House, 2001.

[13] V. Kanodia, A. Sabharwal, E. Knightly, "MOAR: A Multichannel Opportunistic Auto Rate Media Access Protocol for ad hoc networks", Proc. IEEE BROADNETS 2004, Pp. 600-610, October 2004.

[14] H. Zheng, L. Cao, "Device centric spectrum management", in: Proc. IEEE DySPAN 2005, Pp. 56-65, November 2005.

[15] S. Krishnamurthy, M. Thoppian, S. Venkatesan, R. Prakash, "Control channel based MAC-layer configuration, routing and situation awareness for cognitive radio networks", in: Proc. IEEE MILCOM 2005, October 2005. 
[16] V. Kapoor, G. Edwards, and R. Snkar, "Handoff criteria for personal communication networks", Proc. IEEE ICC '94, Pp. 1297-1301, May 1994.

[17] R. Zhang, Y. C. Liang, "Exploiting multi-antennas for opportunistic spectrum sharing in cognitive radio networks," in Proc. IEEE PIMRC, Athens, Greece, Pp. 1-5, Sept. 2007.

[18] ] L. Zhang, Y. C. Liang, and Y. Xin, "Robust cognitive beam forming with partial channel state information," in Proc. CISS 2008, Pp. 890895, Mar. 2008.

[19] M. H. Islam, Y. C. Liang, and A. T. Hoang, "Joint beam forming and power control in the downlink of cognitive radio networks," in Proc. IEEE WCNC, Hong Kong, Pp. 21-26, Mar. 2007.

[20] Karama Hamdi, Wei Zhang and Khaled Ben Letaief, "Opportunistic Spectrum Sharing in Cognitive MIMO Wireless Networks", IEEE TRANSACTIONS ON WIRELESS COMMUNICATIONS, VOL. 8, NO. 8, Pp. 4098-4109, AUGUST 2009.

[21] Sudhir Srinivasa and Syed Ali Jafar, "How Much Spectrum Sharing is Optimal in Cognitive Radio Networks?" IEEE TRANSACTIONS ON WIRELESS COMMUNICATIONS, Vol. 7, NO. 10, Pp. 4010-4018, OCTOBER 2008.

[22] Dusit Niyato and Ekram Hossain, "Competitive Spectrum Sharing in Cognitive Radio. Networks: A Dynamic Game Approach", IEEE TRANSACTIONS ON WIRELESS COMMUNICATIONS, Vol. 7, NO. 7, Pp. 2651-2660, JULY 2008.

[23] Ian F. Akyildiz, Won-Yeol Lee, Mehmet C. Vuran, and Shantidev Mohanty "NeXt generation dynamic spectrum access cognitive radio wireless networks: A survey”. doi:10.1016/j.comnet.2006.05.001.

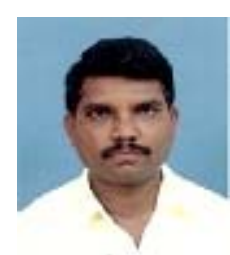

R. Vadivelu is currently working as Assistant Professor in the Department of Electronics and Communication Engineering at Sri Krishna College of Technology, Coimbatore, India. He received his B. Sc., Physics Degree from Bharathiar University, India in 1995, M.Sc., Degree in Electronics Science from University of Madras, India in 1998, M. Tech., Electronics (Microwave and RADAR Systems) from Cochin University of Science and Technology, India in 2001 and pursuing Doctorate Degree in the area of Wireless Communication Technology from Anna University, India. The Author is a life Member of ISTE. He also authored 4 Research Articles in the area of Cognitive Radio in various International Journals. His areas of interest include Spectrum Sensing, RADAR Systems and Microwave Communication. (Email:vadiveluece@gmail.com)

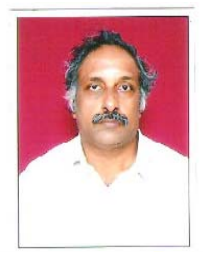

K. Sankaranarayanan, born on 15.06.1952, completed his B.E.(Electronics and Communication Engineering) in 1975, and M.E.(Applied Electronics) in 1978 from P.S.G.College of Technology, Coimbatore under University of Madras. He did his Ph.D. (Biomedical Digital Signal Processing and medical Expert System) in1996 from P.S.G.College of Technology, Coimbatore under Bharathiar University. He has more than 32 years of teaching experience and worked in various Government and self financing Engineering colleges. At present he is working as DEAN at EASA College of Engineering and Technology, Coimbatore, Tamil Nadu, India. His areas of interest include Digital Signal Processing, Computer Networking, Network Security, Biomedical Electronics, Neural Networks and their applications, and Opto Electronics. (E-mail:kkd_sankar@yahoo.com) 